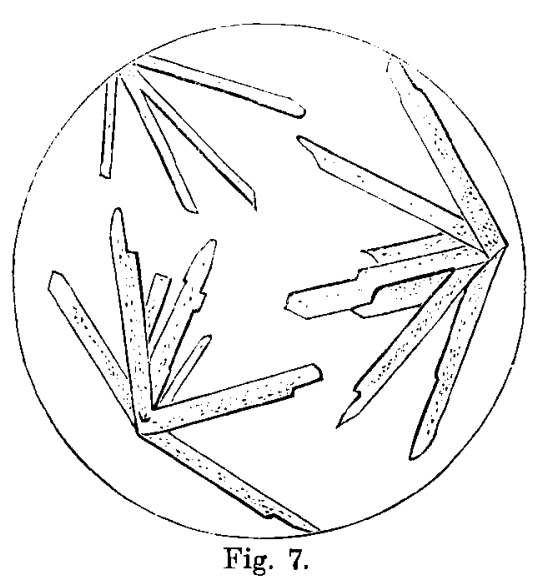

Sie sind optisch positiv. Das Innere der Krystalle erscheint bäufig getrübt durch stabförmige Partikelchen, die sich bei stärkerer Vergrösserung als Gas- und Flüssigkeitseinschlüsse zu erkennen geben.

\title{
Carpaïnpikrat.
}

Zitronengelbe, stark lichtbrechende Kügelchen, welche meist isoliert vorkommen und einen Durchmesser von $0,02-0,04 \mathrm{~mm}$ besitzen. Zwischen gekreuzten Nicols erscheint das stehende Kreuz sphaerolithischer Aggregate. Dieselben sind optisch positiv.

Carpainhydrochlorid und Jod-Jodkalium.

Fügt man zu der alkoholischen Lösung des salzsauren Carpaïns einen Tropfen der Jod-Jodkaliumlösung, so entsteht sofort ein brauner Niederschlag. Derselbe setzt sich bei der Betrachtung unter dem Mikroskop aus orangefarbig durchscheinenden Kügelchen zusammen, die einen Durchmesser von $0,05-0,07 \mathrm{~mm}$ besitzen, sehr stark lichtbrechend und optisch isotrop sind.

Die weiteren Untersuchungen über das Carpaïn werden vorbehalten.

Leiden, April 1897.

Mitteilungen aus dem pharmazeutisch.chemischen Institut der Universität Marburg.

\section{Ueber die Alkaloide der Lupinensamen.}

Von Ernst Schmidt.

(Dritte Mitteilung.) ${ }^{1}$ )

\section{Ueber die Alkaloide der schwarzen Lupine.}

\author{
Von Dr. K. Gerhard.
}

Die schwarze Lupine, deren Namen von der schwarzen Farbe ihrer Samen herrührt, ist eine Spielart von der gewöhnlichen gelben Lupine. Sie ist erst seit einigen Jahren gezuchtet und hat sich als konstant erwiesen.

1) Vergl. dieses Archiv 1897, S. 192 und 262. 
Der Vorzug, den ihr einige Landwirte vor der gelben Lupine geben und daher als Stickstoffsammler und Futtermittel verwenden, soll nach Kette ${ }^{1}$ ) darin bestehen, dass sie "weniger empfindlich gegen Mergel im Boden und der gelben Lupine im Massenertrage überlegen ist". Weitere Angaben über die Vorzüge, welche dieselbe bei dem relativ hohen Preise für landwirtschaftliche Zwecke besitzt, habe ich nicht erhalten können.

Um über die Pflanze selbst etwas zu erfahren und eventuelle Unterschiede von der gelben Lupine festzustellen, wurden im Mai einige Samen der schwarzen zugleich mit denen der gelben Lupine auf mittelschwerem Gartenboden gesäet, beide Pflanzen dann in den verschiedenen Wachstumsstadien untersucht und miteinander verglichen.

Ende Juli kamen beide Arten fast zu gleicher Zeit zur Blüte, während ihre Samen erst Anfang September zur Reife gelangten.

Bei den angestellten Untersuchungen über die Morphologie der Pflanzen stellten sich die Färbung und die Gestalt der Samen als einziger Unterschied heraus: während die Samen der gelben Lupine von mehr walzenförmiger Gestalt sind und hellgelb, schwarz punktiert erscheinen, sind die schwarzen Lupinensamen mehr oder weniger plattgedrückt und gleichmässig schwarz gefärbt, mit einem hellgelben Bande gezeichnet, welches an der Ecke, wo sich der Nabel befindet, im Bogen über den Samen verläuft.

Anatomisch erwiesen sich beide Pflanzen ebenfalls als gleich, mit dem einzigen Unterschiede, dass der Farbstoff bei den Samen der gelben Art ungleichmässig, bei denen der schwarzen Lupine gleichmässig in den unter der Kutikula liegenden, stark verdickten, pallisadenartigen Zellen verteilt liegt und so die verschiedene Färbung der Samen bedingt.

Quantitative Alkaloidbestimmung.

Bevor ich damit begann, die Samen der schwarzen Lupine zu verarbeiten, führte ich von einer Probe eine quantitative Alkaloidbestimmung aus, um einen ungefähren Anhalt über die zu erwartende Ausbeute zu erhalten. Ich verfuhr zu diesem Zwecke nach der von Keller empfoblenen Methode in folgender Weise:

15,0 mittelfeines, bei $30^{\circ}$ getrocknetes Samenpulver wurden mit einem Gemisch aus 100,0 Aether, 50,0 Chloroform und $10 \mathrm{~g} 10 \%$ igem Salmiakgeistes eine halbe Stunde lang geschüttelt, absetzen lassen und filtriert. Vou 100,0 Filtrat $(=10,0$ Samen) wurde das Aether-Chloroformgemisch im Dampfbade abdestilliert, der gelbe Rückstand mit einigen Tropfen verdünnter Salzsäure angesäuert, einige Kubikzentimeter Wasser hinzugefügt, diese salzsaure Lösung durch ein kleines Filter filtriert und Kolben und Filter gut nachgewaschen.

1) Kette, Lupinenbau, S. 104. 
Das Filtrat, aufs neue mit Ammoniak alkalisch gemacht, wurde darauf mit 50,0 Aether und 25,0 Chloroform eine halbe Stunde lang geschüttelt, von 60,0 Filtrat ( $=8,0$ Samen) das Aether-Chloroformgemisch abdestelliert (wobei die Vorsicht gebraucht wurde, die letzten Kubikzentimeter unter Umschwenken

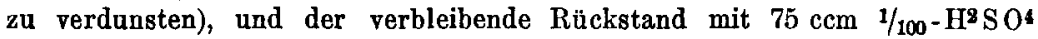
digeriert. Das Filtrat mitsamt dem Waschwasser wurden in eine $200 \mathrm{ccm}$ Stöpselflasche gethan, mit einer $2 \mathrm{ccm}$ hohen Schicht Aether überschichtet und mit zwei Tropfen ätherischer Jodeosinlösung versetzt.

Das Zurücktitrieren der überschüssigen $1 / 100-\mathrm{H}^{2} \mathrm{SO}^{4}$ geschah in der Weise, dass ich aus einer Bürette kubikzentimeterweise soviel $1 / 100-\mathrm{N} \cdot \mathrm{KOH}$ hinzufliessen liess, bis beim kräftigen Durchschütteln der Flüssigkeit und jedesmaligen Absetzen- und Sichtrennenlassen der Schichten, die untere, wässerige Schicht anfing, sich schwach rosa $z u$ färben, und der darauf schwimmende Aether farblos geworden war. Dieses war nach Zusatz. von $54,5 \mathrm{ccm} \mathrm{1/100}-\mathrm{N} \cdot \mathrm{KOH}$ erreicht, mithin waren zur Bindung der freien Base (aus 8,0 Samen) $20,5 \mathrm{ccm} 3 / 100^{-} \mathrm{H}^{2} \mathrm{~S} \mathrm{O}^{4}$ verbraucht, die, auf Lupanin, $\mathrm{C}^{15} \mathrm{H}^{24} \mathrm{~N}^{2} \mathrm{O}$, berechnet, einem Gehalt von $0,61008_{o}^{\%}$ entsprachen, oder, auf Lupinin, $\mathrm{C}^{21} \mathrm{H}^{40} \mathrm{~N}^{2} \mathrm{O}^{2}$, bezogen, $0,86592 \%$ ergaben.

Da die Litteraturangaben über den Alkaloidgehalt der verschiedenen Lupinensorten sehr von einander abweichen, so führte ich nach der soeben angegebenen Keller'schen Methode ausserdem Alkaloidbestimmungen von den Samen der gelben, blauen, weissen und perennierenden Lupine, welche von Haage \& Schmidt, Erfurt, bezogen waren, aus. Die Bestimmungen ergaben folgende Resultate:

Die aus je 100,0 Samen gewonnenen Alkaloide erforderten zur Neutralisation:

$$
\begin{gathered}
\text { bezog. auf } \\
\text { Lupanin } \\
\text { Lupog. auf } \\
\mathrm{C}^{15} \mathrm{H}^{24} \mathrm{~N}^{2} \mathrm{O} \mathrm{C}^{21} \mathrm{H}^{40} \mathrm{~N}^{2} \mathrm{O}^{2}
\end{gathered}
$$

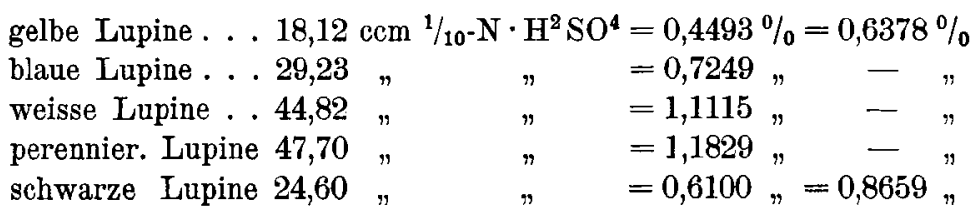

$\mathrm{Da}$, wie sich später herausstellte, die schwarzen Lupinen ausser Lupinin, noch Lupinidin, $\mathrm{C}^{8} \mathrm{H}^{15} \mathrm{~N}$, dessen Gehalt nicht genau zu ermitteln ist, enthalten, hat man es bei dieser Alkaloidbestimmung nur mit annähernden und zwar relativen Werten zu thun.

Darstellung der Alkaloide aus den Samen der schwarzen Lupine.

Die Samen, welche in einer Menge von $25 \mathrm{~kg}$ fur die Extraktion der Alkaloide benutzt wurden, stammten aus der Samenhandlung von Haage \& Schmidt in Erfurt. 
Die Verarbeitung dieser Samen auf Alkaloide geschah in der Weise, wie es früher für die Samen der blauen, weissen und gelben Lupinen beschrieben ist (s. dieses Archiv 1897, 197 u. 263). Nach dem Abdestillieren des Chloroforms restierte ein rotgelbes Liquidum von intensiv bitterem Geschmack und honigartigem Geruch. Dieses Chloroformextrakt liess ich zunächst 14 Tage im Exsiccator über $\mathrm{H}^{2} \mathrm{SO}^{4}$ stehen; hierdurch färbte sich die Rohbase noch dunkler, ohne dass jedoch eine Krystallbildung eintrat. Zur weiteren Reinigung dieser Rohbasen nahm ich daher eine Probe derselben mit Wasser auf, versetzte diese Lösung mit einem Ueberschuss von Bleiacetat und liess nach gutem Durchschütteln den entstandenen Niederschlag sich absetzen; alsdann filtrierte ich denselben $a b$, wusch ihn gut aus und befreite das Filtrat durch $\mathrm{H}^{2} \mathrm{~S}$ von dem überschüssigen Blei. Die noch immer stark gelb gefärbte, von $\mathrm{PbS}$ befreite Lösung wurde hierauf mit frisch ausgeglühter Tierkohle behandelt, um die letzten Verunreinigungen noch zu entfernen.

Die nach dem Abfiltrieren und Auswaschen erhaltene Flüssigkeit, die nur noch schwach gelb gefärbt war, wurde mit Salzsäure angesäuert, stark eingedickt und nach Zusatz von überschüssiger Natronlauge von neuem mit Chloroform ausgeschüttelt. Es hinterblieb nach dem Abdestillieren des Chloroforms ein gelb gefärbtes, dickes Liquidum, welches selbst ṇach längerem Stehen über Schwefelsäure ebenfalls nicht krystallisierte.

Ich versuchte daher eine Reinigung des Rohalkaloids dadurch zu erreichen, dass ich dasselbe in wenig Wasser löste, mit $\mathrm{H} \mathrm{Cl}$ schwach ansäuerte, mit konzentrierter Natronlauge wieder alkalisch machte und diese Mischung mit Aether ausschüttelte. Da die Alkaloide von diesem Lösungsmittel nur sehr schwer aufgenommen wurden, musste ich das Ausschütteln längere Zeit fortsetzen. Ich erzielte hierdurch eine relativ sehr reine Alkaloidlösung, die nach dem Verdunsten des Aethers ein nur schwach gelblich gefärbtes Liquidum hinterliess. Dieses wurde in einer Krystallisierschale einige Tage im Exsiccator stehen gelassen, wodurch sich am Boden der Schale eine grosse Menge sehr schön ausgebildeter, weisser, durchsichtiger, rhombischer Krystalle abschied, die, wie die weitere Untersuchung ergab, sich als Lupinin erwiesen.

\section{Lupinin.}

Behufs weiterer Reinigung trennte ich die nach obigen Angaben abgeschiedenen Krystalle durch Absaugen von der Mutterlange, spülte sie mit Alkohol und Aether ab und krystallisierte sie ans Petroleumäther so oft um, bis rein weisse, an der Luft sich nicht mehr färbende Krystalle resultierten. Die aus der Mutterlauge des Alkaloidgemisches sich im Laufe der Zeit abscheidenden Krystalle wurden in gleicher Weise gereinigt. Ich erhielt auf diese Weise etwa 25,0 reiner Base, 
die ich für die weiteren Untersuchungen verwendete. Die letzte Mutter. lauge liess ich noch etwa acht Wochen zum Krystallisieren im Exsiccator stehen; es fand indessen keine weitere Abscheidung von Krystallen statt, obwohl, wie die spätere Untersuchung zeigte, noch reichliche Mengen Lupinins darin vorhanden waren.

Ueber die Trennung und Isolierung des Lupinidins aus dem Alkaloidgemenge werde ich weiter unten berichten.

Das von mir aus den Samen der schwarzen Lupine isolierte, krystallinische Alkaloid stimmte in seinen Eigenschaften durchaus mit den Angaben überein, die Baumert ${ }^{1}$ ) und Berend ${ }^{2}$ ) über das Lupinin der gelben Lupine machen.

Je nach der Konzentration der Lösung erhielt ich strahligkrystallinische, weisse Drusen, durchsichtige farblose Tafeln oder lange, glashelle, säulenförmige Krystalle, die alle dem rhombischen Krystallsystem angehörten.

Den Schmelzpunkt der aus Petroleumäther auskrystallisierten Base fand ich stets bei 68 bis $69^{\circ} \mathrm{C}$. liegend, während Baumert denselben für das aus den gelben Lupinen isolierte Lupinin bei 67,5 bis $68,5^{\circ} \mathrm{C}$., Berend bei 67 bis $68^{\circ} \mathrm{C}$. fand.

Abweichend von Baumert, konnte ich jedoch eine Reduktion der Fehling'schen Lösung nicht wahrnehmen.

Um das spezifische Drehungsvermögen des aus den Samen der schwarzen Lupine isolirten Lupinins zu bestimmen, löste ich 0,4104 reiner, trockener Base in 11,3561 Wasser. Das spezifische Gewicht der Lösung betrug 1,005 bei $15^{\circ} \mathrm{C}$. Der polarisierte Lichtstrahl wurde bei einer $2 \mathrm{dm}$ langen Flüssigkeitssäule um $1^{0} 22^{\prime}$ (im Mittel) nach links abgelenkt. Die Temperatur betrug $17^{\circ} \mathrm{C}$.

Demnach ergiebt sich für

$$
[a] \mathrm{D}-19^{\circ} \text { (bei } 17^{\circ} \mathrm{C} \text {.). }
$$

Die Verbrennungen der im Exsiccator getrockneten Base lieferten folgende Zahlen:

1. 0,1708 Substanz ergaben $0,4486 \mathrm{CO}^{2}$ und $0,1797 \mathrm{H}^{2} 0$,

2. $0,2499 " n 0,6569 \mathrm{C} \mathrm{O}^{2}$ n $0,2651 \mathrm{H}^{2} \mathrm{O}$. Gefunden:

I. II.

C . . $71,63 \%$. $71,68 \%$.

$\mathrm{H}$. . . 11,68, 11,78,

Berechnet für Baumert Berend

$\mathrm{C}^{21} \mathrm{H}^{40} \mathrm{~N}^{2} \mathrm{O}^{3}$ gefunden gefunden

$\mathrm{C} \cdot \cdot \cdot \cdot \cdot 71,59 \%$.

$71,51 \%$. $\quad 71,42 \%$.

11,72 ,

Gerhard

gefunden

$71,65 \%$.

H... . . 11,36,

11,61 ,

11,73 ,

1) Habilitationsschrift "Lupinin“, S. 12-16.

2) Dieses Archiv, 1897, S. 26 כ. 
Auf Grund der mit dem Lupinin übereinstimmenden Eigenschaften und der Werte, die ich beim Verbrennen meiner Base erhalten habe, glaube ich annehmen zu dürfen, dass das von mir aus den schwarzen Lupinensamen isolierte, krystallinische Alkaloid mit dem Lupinin der gelben Lupine identisch ist, und ihm die Baumertsche Formel $\mathrm{C}^{21} \mathrm{H}^{40} \mathrm{~N}^{2} \mathrm{O}^{2}$ zuzuerteilen ist. Als Stütze für diese Annahme und zur weiteren Charakterisierung der Base als Lupinin, titrierte ich dieselbe mit $1 / 100-\mathrm{N} \cdot \mathrm{H}^{2} \mathrm{SO}^{4}$, unter Anwendung von Jodeosin als Indikator, und stellte ausserdem noch einige Salze dar, welche ich analysierte.

Lupininplatinchlorid: $\mathrm{C}^{21} \mathrm{H}^{40} \mathrm{~N}^{2} \mathrm{O}^{2} \cdot 2 \mathrm{HCl} \cdot \mathrm{PtCl}^{4}+\mathrm{H}^{2} \mathrm{O}$. Zur Darstellung dieses Salzes löste ich etwa 3,0 reines Lupinin in Wasser, versetzte diese Lösung mit Platinchloridlösung, nachdem ich sie zuvor mit $\mathrm{HCl}$ angesäuert hatte, und liess sie im Exsiccator freiwillig verdunsten. Nach einigen Tagen schieden sich rubinrote, harte, durchsichtige Krystalle ab, die dem klinorhombischen System angehörten. Das so gewonnene Platinsalz krystallisierte aus wässerigen und alkoholischen Lösungen immer in denselben Krystallformen. Es war leicht löslich in heissem Wasser und Alkohol. Beim Liegen an der Luft erwies es sich als sehr beständig, es bewahrte seine Durchsichtigkeit und zeigte keine Neigung zum Verwittern.

Den Schmelzpunkt des lufttrockenen Salzes fand ich, in Uebereinstimmung mit Liebscher, bei $165^{\circ} \mathrm{C}$. liegend. Berend fand 163 bis $164^{\circ} \mathrm{C}$.

Im Exsiccator verlor das Platinsalz schon kleine Mengen Wasser; beim Trocknen bei $100^{\circ}$ gab es sein gesamtes Krystallwasser ab:

\begin{tabular}{|c|c|c|c|c|}
\hline & 0,2076 & Substanz & verloren & 0 \\
\hline II. & 0,1755 & & 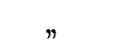 & 0,0040 \\
\hline & 0,1406 & $n$ & & 0,0033 \\
\hline & 0,2063 & $n$ & 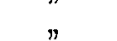 & 0,0048 \\
\hline
\end{tabular}

I. 0,2028 bei $100^{0}$ getrockneter Substanz lieferten 0,0524 Pt II. 0,1721 III. 0,1373 "

" $\quad " \quad$ " $\quad 0,0438$,

0,2015 bei $100^{\circ}$ getrock. Substanz lieferten $0,1050 \mathrm{H}^{2} \mathrm{O}$ und $0,2431 \mathrm{CO}^{2}$.

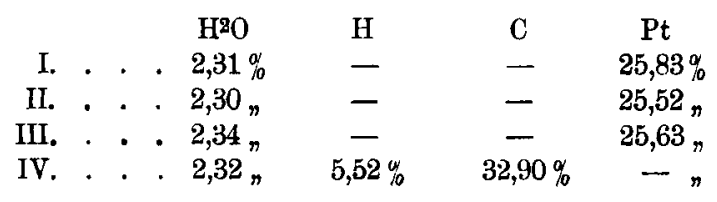




\begin{tabular}{|c|c|c|c|c|c|}
\hline & & Baumert & Berend & $\begin{array}{c}\text { berechnet für } \\
\mathrm{C}^{21} \mathrm{H}^{40} \mathrm{~N}^{2} \mathrm{O}^{2} \cdot 2 \mathrm{HCl}\end{array}$ & Gerhard \\
\hline & & gefunden & gefunden & - $\mathrm{PtCl}^{4}+\mathrm{H}^{2} \mathrm{O}$ & gefunden \\
\hline $\mathrm{Pt}$ & . & . $\quad 25,84 \%$ & $25,66 \%$ & $25,54 \%$ & $25,66 \%$ \\
\hline $\mathrm{C}$ & . & . 32,78 & 32,95 " & 33,09 , & 32,90 " \\
\hline $\mathrm{H}$ & . & 5,69 & 5,81, & 5,51, & 5,52, \\
\hline $\mathrm{H}^{2} \mathrm{O}$ & . & . $\quad 2,27$, & 2,30 & 2,30 & 2,31, \\
\hline
\end{tabular}

Lupiningoldehlorid: $\mathrm{C}^{21} \mathrm{H}^{40} \mathrm{~N}^{2} \mathrm{O}^{2} \cdot 2 \mathrm{HCl} \cdot 2 \mathrm{AuCl}^{3}$. Zur Darstellung dieses Salzes löste ich ca. 2,0 reines Lupinin in salzsäurehaltigem Wasser und versetzte diese Lösung so lange mit Goldchlorid, bis keine Fällung mehr erfolgte. Den entstandenen hellgelben, flockigen Niederschlag liess ich gut absetzen, sammelte ihn auf einem Filter, wusch ihn mit wenig Wasser aus und löste ihn in viel salzsäurehaltigem, heissem Wasser wieder auf. Diese Lösung liess ich über Schwefelsäure verdunsten. Nach einigen Tagen hatte sich das Salz in schönen, gelben, nadelförmigen, federbartartig aneinander gereihten Krystallen abgeschieden.

Dieselben erwiesen sich als leicht löslich in heissem Wasser und Alkohol, wohingegen kaltes Wasser nur wenig von dem Goldsalz zu lösen vermochte.

Aus der alkoholischen Lösung schieden sich schöne, durchsichtige Blättchen $a b$, die indessen nur schwer frei von metallischem Gold zu erhalten waren.

Der Schmelzpunkt war kein scharfer; ich fand ihn zwischen $211^{\circ}$ und $215^{\circ} \mathrm{C}$. liegend, während das von Baumert beschriebene Salz bei ca. $195^{\circ} \mathrm{C}$, das von Berend beschriebene, bei $196-197^{\circ} \mathrm{C}$. schmolz. Das erhaltene Goldsalz erwies sich als wasserfrei.

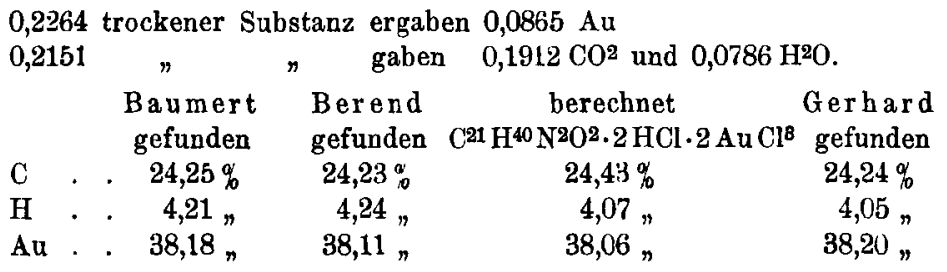

Salzsaures Lupinin: $\mathrm{C}^{21} \mathrm{H}^{40} \mathrm{~N}^{2} \mathrm{O}^{2} \cdot 2 \mathrm{HCl}$. Die Darstellung des salzsauren Lupinins führte ich in der Weise aus, dass ich ca. 3,0 reines, krystallisiertes Lupinin in wenig Wasser löste, diese Lösung mit verdünnter Salzsäure genau neutralisierte und auf dem Dampfbade zum dicken Sirup eindampfte. Derselbe gestand zu einer dicken, zähflüssigen, federbartartig krystallinischen Masse, die ich nach den Angaben Baumert's abwechselnd so lange mit. absolutem Alkohol aufnahm und letzteren auf dem Dampfbade wieder verdunstete, bis ein 
von Baumert ${ }^{1}$ ) zur Trennung von Lupinin und Lupinidin benutzte Verfahren mit gutem Erfolg auch zur Scheidung der in den schwarzen Lupinensamen enthaltenen Basen an. Dasselbe beruht auf dem Umstand, dass das Lupinidin mit Schwefelsäure, bei geeigneter Behandlung mit absolutem Alkohol, ein in letzterem schwer lösliches saures Salz von sandiger Beschaffenheit bildet, während das saure Lupininsulfat ein in absolutem Alkohol sehr leicht löslicher Sirup ist, der nur äusserst schwer krystallinisch wird.

Dieses von Baumert empfohlene Trennungsverfahren hat vor dem Beyerschen den Vorteil, dass aus dem erhaltenen, sauren schwefelsauren Lupinidin die freie Base viel leichter mittelst Natronlauge zu isolieren ist, als dieses durch Zerlegung des sehr schwer löslichen Platindoppelsalzes möglich ist.

Lupinidinplatinchlorid: $\mathrm{C}^{16} \mathrm{H}^{32} \mathrm{~N}^{2} \mathrm{PtCl}^{8}+2 \mathrm{H}^{2} \mathrm{O}$. Das nach dem Beyerschen Verfahręn aus dem sirupösen Alkaloid-Rückstande durch Platinchlorid ausgefälite und aus heissem Wasser umkrystallisierte Platinsalz lieferte, je nach der Konzentration der Lösung, bald grosse, dunkelrote Krystalle, oder glänzende, harte, orangefarbene Nadeln, zu Drusen gruppiert, bald hellere, mattglänzende, feine Nadeln oder endlich ein aus gelben, weichen Schuppen bestehendes Krystallmehl. Alle diese Krystallformen gehörten dem rhombischen System an.

Das Platinsalz war in heissem Wasser schwer löslich, leichter dagegen in salzsäurehaltigem, heissem Wasser. In kaltem Wasser und Alkohol war es fast unlöslich. Sämtliche Krystallisationen verhielten sich beim Erhitzen im Schmelzpunktröhrchen übereinstimmend: es trat zwischen $285^{\circ}$ und $240^{\circ} \mathrm{C}$. Verkohlung ein ohne vorheriges Schmelzen.

Beim Trocknen bei $100^{\circ}$ verlor das Salz nur wenig an Gewicht; erst über $100^{\circ}$ getrocknet, gab es sämtliches Krystallwasser ab, und zwar wurde bei $135^{\circ}$ der Gewichtsverlust konstant.

Die Gewichtsabnahme des bei $135^{\circ}$ bis zum konstantem Gewicht getrockneten Salzes betrug von:
I. 0,1762 Substanz $0,0098 \mathrm{H}^{2} \mathrm{O}$
II. $0,2364 \quad " 0,0133$ "
III. $0,2297 " 0,0131 "$

I. 0,2179 bei $135^{\circ}$ getrockneter Substanz ergaben $0,0648 \mathrm{Pt}=29,74 \% \mathrm{Pt}$

II. $0,1506 " n \quad " \quad " \quad n \quad 0,0444,=29,48, "$

III. $0,2231 " \pi \quad " \quad " \quad$ " $" 0,0661 "=29,62 " n$

I. 0,2331 lufttrockener Substanz lieferten $0,0982 \mathrm{H}^{2} \mathrm{O}$ und $0,2312 \mathrm{CO}^{2}$

II. $0,1965 \quad " \quad$ " 0,0802 " 0,1991 ,

1) Liebig's Annalen 225, S. 368. 
K. Gerhard: Alkaloide der schwarzen Lupine.

Gefunden für bei $135^{\circ}$ getrockneter Substanz:

I.

II.

III.

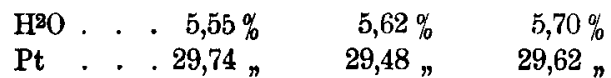

Gefunden für lufttrockene Substanz:

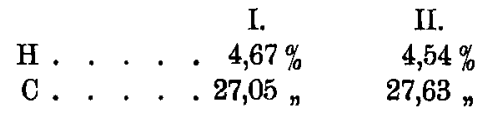

Bezogen und umgerechnet auf lufttrockene Substanz, ergeben sich hieraus folgende Daten:

\begin{tabular}{|c|c|c|c|}
\hline $\begin{array}{l}\text { Baumert } \\
\text { gefunden }\end{array}$ & $\begin{array}{l}\text { Berend } \\
\text { gefunden }\end{array}$ & $\begin{array}{c}\text { berechnet für } \\
\mathrm{C}^{16} \mathrm{H}^{82} \mathrm{~N}^{2} \mathrm{P} t \mathrm{Cl}^{6}+2 \mathrm{H}^{2} \mathrm{O}\end{array}$ & $\begin{array}{l}\text { Gerhard } \\
\text { gefunden }\end{array}$ \\
\hline $27,15 \%$ & $27,80 \%$ & $27,60_{10}^{\circ}$ & $27,34 \%$ \\
\hline $5,34 n$ & $5,13 "$ & 5,17 & 4,60 , \\
\hline Pt 28,39, & 27,91 & $27,96 n$ & 28,08, \\
\hline $\mathrm{H}^{2} \mathrm{O} \quad 5,32_{n}$ & $5,19 "$ & $5,17 n$ & 5,62 \\
\hline
\end{tabular}

Aus den Eigenschaften und den analytischen Daten, welche ich für das vorliegende Platinsalz der schwarzen Lupine erhalten habe, geht zur Genüge hervor, dass dieses Platinsalz mit dem Lupinidinplatinchlorid der gelben Lupine identisch und ihm die von Baumert aufgestellte Formel $\mathrm{C}^{16} \mathrm{H}^{32} \mathrm{~N}^{2} \mathrm{Pt} \mathrm{Cl}^{6}+2 \mathrm{H}^{2} \mathrm{O} \mathrm{zu}$ zuerteilen ist.

Zur weiteren Charakterisierung des Lupinidins der schwarzen Lupine stellte ich noch einige andere Salze von demselben her.

Saures schwefelsaures Lupinidin: $\mathrm{C}^{8} \mathrm{H}^{15} \mathrm{~N} \cdot \mathrm{H}^{2} \mathrm{SO}^{4}$. Die Gewinnung dieses Salzes aus den Rohalkaloiden nach der Baumertschen Methode ist bereits oben angegeben worden. Die weitere Reinigung geschah in der Weise, dass ich das zunächst erhaltene, gelblichgraue Pulver in so viel Wasser zerfliessen liess, als eben zur Lösung erforderlich war, und zu dem auf diese Weise resultierenden Sirup unter Umschwenken successive die vierfache Menge absoluten Alkohols hinzufügte. Es schied sich dadurch sofort ein rein weisses, klein krystallinisches Mehl ab, welches nach dem Auswaschen mit absolutem Alkohol und Trocknen zwischen Fliesspapier über Schwefelsäure aufbewahrt wurde.

Dieses so erhaltene Sulfat war in Wasser äusserst leicht löslich, dagegen fast unlöslich in absolutem Alkohol. Die Lösung dieses Salzes zeigte stark saure Reaktion und drehte die Schwingungsebene des polarisierten Lichtes nach links. Bei $100^{\circ}$ getrocknet, verlor es. nichts an Gewicht.

Das bei $100^{\circ}$ getrocknete Salz schmolz unter Zersetzung und Gasentwicklung bei $242^{\circ} \mathrm{C}$. 
0,1944 trockener Substanz ergaben 0,2030 Ba S04 $=43,04 \% \mathrm{SO}^{4}$.

$\begin{array}{ccc}\text { Ba amert } & \text { berechnet } & \text { Gerhard } \\ \text { gefunden } & \mathrm{C}^{8} \mathrm{H}^{15} \mathrm{~N} \cdot \mathrm{H}^{2} \mathrm{SO}^{4} & \text { gefunden } \\ \mathrm{SO}^{4} 43,35 \% & 43,05 \% & 43,04 \%\end{array}$

Um das spezifische Drehungsvermögen zu bestimmen, löste ich 0,4072 bei $100^{\circ}$ getrockneten Salzes in 18,0130 Wasser bei $17^{\circ}$. Das spezifische Gewicht der Lösung betrug 1,0082. Der polarisierte Lichtstrahl wurde bei einer $2 \mathrm{dm}$ langen Flüssigkeitssäule um 42' (im Mittel) nach links abgelenkt. Als Lichtquelle wurde die Chlornatriumflamme benutzt. Das spezifische Drehungsvermögen beträgt demnach, hierauf berechnet:

$$
[\alpha]_{D}=-15,33^{0} .
$$

Jodwasserstoffsaure Salze des Lupinidins.

Das Lupinidin verbindet sich, wie die Arbeiten von Baumert und Berend lehren, in charakteristischer Weise mit Jodwasserstoff in zwei verschiedenen Verhältnissen, indem sich $1 \mathrm{Mol}$. Base mit $1 \mathrm{Mol}$. HJ, sowie auch $2 \mathrm{Mol}$. Base mit $1 \mathrm{Mol}$. HJ vereinigen. Es schien daher von Interesse zu sein, das Lupinidin der schwarzen Lupine auch nach dieser Richtung hin zu untersuchen.

a) Neutrales jodwasserstoffsaures Lupinidin: $\mathrm{C}^{8} \mathrm{H}^{15} \mathrm{~N} \cdot \mathrm{HJ}+1 / 2$ $\mathrm{H}^{2} \mathrm{O}$. Zur Gewinnung dieses Salzes löste ich das entsprechende Hydrochlorid (aus dem Platinsalz dargestellt) in wenig Wasser auf und fügte eine konzentrierte, wässerige Jodkaliumlösung hinzu. Es schied sich sofort ein rötlich weisser, amorpher Niederschlag aus, der sich in warmem Wasser leicht auflöste und bei rascher Abkühlung in kleinen, weissen Blättchen wieder zur Abscheidung gelangte. Bei langsamer Abkühlung und freiwilligem Verdunstenlassen dieser Lösung schieden sich grosse, weisse, durchsichtige Krystalle ab, die beim Liegen an der Luft schnell gelb und undurchsichtig wurden. Mit den hellen, weissen Krystallen schieden sich nicht selten stark gefärbte Perjodide ab; man thut daher gut, zu der Salzlösung etwas Alkohol hinzuzufügen, welcher die Perjodide in Lösung hält. Durch öfteres Umkrystallisieren erhielt ich völlig farblose, durchsichtige, wohlausgebildete Krystalle, die an der Luft stets ihre Durchsichtigkeit einbüssten und nach einiger Zeit schwach gelb gefärbt wurden. Dieselben zeigten stark saure Reaktion.

Der Schmelzpunkt dieses lufttrocknen jodwasserstoffsauren Salzes war nicht scharf, er lag zwischen 245 und $257^{\circ} \mathrm{C}$.

Beim Trocknen bei $100^{\circ}$ verloren:

0,1926 Substanz $0,0062 \mathrm{H}^{2} \mathrm{O}$.

$0,186+$ bei $100^{\circ}$ getrockneter Substanz lieferten 0,1722 AgJ. 
K. Gerhard: Alkaloide der schwarzen Lupine.

$\begin{array}{cccc}\begin{array}{c}\text { Baumert } \\ \text { gefunden }\end{array} & \begin{array}{c}\text { Berend } \\ \text { gefunden }\end{array} & \begin{array}{c}\text { berechnet für } \\ \mathrm{C}^{8} \mathrm{H}^{15} \mathrm{~N} \cdot \mathrm{HJ}+1 / 2 \mathrm{H}^{2} \mathrm{O}\end{array} & \begin{array}{c}\text { Gerhard } \\ \text { gefunden }\end{array} \\ \mathrm{J} \quad 50,36 \% & 50,27 \% & 50,19 \% & 49,92 \% \\ 1 / 2 \mathrm{H}^{20} 3,12 \% & 3,06 \% & 3,43 \% & 3,22 \%\end{array}$

b) Basisch jodwasserstoffsaures Lupinidin: $\left(\mathrm{C}^{8} \mathrm{H}^{15} \mathrm{~N}\right)^{2} \cdot \mathrm{HJ}$. Dieses Salz stellte ich in der Weise her, dass ich zunächst aus dem sauren schwefelsauren Lupinidin, nach dem Versetzen mit Natronlauge, die freie Base mit Aether ausschüttelte und dieselbe mit wässeriger, farbloser Jodwasserstoffsäure bis zur schwach sauren Reaktion versetzte. Beim Stehen im Exsiccator schieden sich nach einiger Zeit fast farblose, sehr schön ausgebildete, durchsichtige, harte, glänzende, rhombische Prismen aus, die ich bei mehrfachem Umkrystallisieren ganz farblos erhielt. Dieselben lösen sich leicht in Wasser und Alkohol, bleiben beim Liegen an der Luft durchsichtig und färben sich allmählich gelb. Beim Erhitzen auf $105^{\circ}$ verlieren sie nichts an Gewicht. Der Schmelz. punkt ist scharf und liegt in Uebereinstimmung mit den Angaben von Berend bei 229 bis $230^{\circ} \mathrm{C}$.

0,1834 trockener Substanz lieferten 0,1148 Ag J.

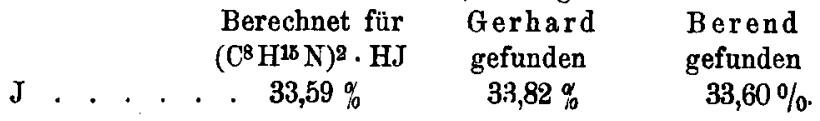

Lupinidinquecksilberchlorid: $\mathrm{C}^{18} \mathrm{H}^{80} \mathrm{~N}^{2} \cdot 2 \mathrm{HCl} \cdot \mathrm{Hg} \mathrm{Cl}^{2}$. Dieses Salz, welches nach Angaben von Berend für Lupinidin besonders charakteristisch ist, erhielt ich, indem ich eine mässig verdünnte Lupinidinchloridlösung, welche ich vorher mit $\mathrm{HCl}$ ansăuerte, mit einer konzentrierten Quecksilberchloridlösung im Ueberschuss versetzte. Es schied sich momentan ein rein weisser, krystallinischer Niederschlag aus, der sich beim Stehen noch vermehrte. Denselben sammelte ich auf einem Filter, wusch ihn mit Wasser aus und kry. stallisierte ihn aus heissem Wasser um. Bei langsamem Erkaltenlassen schieden sich schöne, weisse, durchsichtige, federbartartig gruppierte Krystalle ab. Dieselben waren schwer löslich in kaltem, leichter löslich in heissem Wasser und Alkohol. Sie schmolzen bei $214^{\circ} \mathrm{C}$. unter Zersetzung und Gasentwickelung.

Bei $100^{\circ}$ getrocknet, nahm dieses Quecksilberdoppelsalz nichts an Gewicht ab.

0,2608 trockener Substanz ergaben 0,1022 $\mathrm{Hg} \mathrm{S}$ und 0,2524 $\mathrm{Ag} \mathrm{Cl}$.

Nach diesen analytischen Daten glaube ich annehmen zu dürfen, dass dem Lupinidinquecksilberchlorid, entsprechend den Angaben von Berend, die Formel $\mathrm{C}^{16} \mathrm{H}^{80} \mathrm{~N}^{2} \cdot 2 \mathrm{HCl} \cdot \mathrm{HgCl}^{2}$ zukommt:

$$
\begin{aligned}
& \text { Berechnet } \\
& \mathrm{Hg} \text {. . . . . . . . 33,67\% } 33,78 \% \\
& \mathrm{Cl} \text {. . . . . . . . 23,90 " } 23,94 \text { ” }
\end{aligned}
$$

Arch. d. Pharm. GCXXXV, Bds. 5. Heft. 


\section{Freie Base.}

Die freie Base erhielt ich als ein dickes, hellgelbes, nur schwach riechendes Liquidum, indem ich das saure schwefelsaure oder salzsaure Lupinidin in wenig Wasser löste, mit Natronlauge alkalisch machte, alsdann mit Aether ausschüttelte und letzteren freiwillig verdunsten liess. Der zurückbleibende dicke, stark alkalisch reagierende Sirup wurde selbst bei langem Stehen über Schwefelsäure nicht krystallinisch; er färbte sich sehr schnell dunkler und nahm einen unangenehmen, widerlichen Geruch an. In diesem Zustande löste sich die Base leicht in Chloroform und Alkohol, dagegen schwer in Aether und fast gar nicht in Wasser.

Auch aus diesen Eigenschaften der freien Base geht zur Genüge die Identität des von mir aus den Samen der schwarzen Lupine isolierten, flüssigen Alkaloides mit dem in den gelben Lupinen entbaltenen Lupinidin hervor.

Um festzustellen, ob neben Lupinin und Lupinidin noch andere Alkaloide in den Samen der schwarzen Lupine enthalten seien, liess ich die durch Auskrystallisierenlassen von Lupinin und durch Ausfällen mittels Platinchlorid von Lupinidin grösstenteils befreite Mutterlauge des Basengemisches, nach Zusatz neuer Mengen von Platinchlorid im Exsiccator verdunsten. Aus dieser, der zweiten und dritten Mutterlauge schied sich nur Lupinidinplatinchlorid aus, welches ich durch Schmelzpunkt und Platinbestimmung als solches charakterisierte. Beim Verdunsten der vierten Platinsalzmutterlauge schied sich ein Gemisch zweier Platinsalze aus, die sich indessen mechanisch leicht von einander treunen liessen und als Lupinidin- und Lupininplatinchlorid identifiziert werden konnten.

Nach dem Abfiltrieren dieser Ausscheidungen und weiterem Verdunstenlassen dieser und der folgenden Mutterlaugen gelangte immer nur das einheitliche Platinsalz des Lupinins zur Ausscheidung.

Es ist somit der Beweis erbracht, dass die schwarzen Lupinensamen neben Lupinin und Lupinidin kein drittes Alkaloid, wenigstens nicbt in greifbarer Menge, enthalten.

Aus diesen Untersuchungen ergeben sich daher folgende Resultate:

1. In den Samen der schwarzen Lupine sind zwei Alkaloide enthalten, ein festes und ein flüssiges.

2. Das aus diesen Samen isolierte feste Alkaloid ist identisch mit dem Lupinin der gelben Lupinensamen.

3. Das flussige Alkaloid der schwarzen Lupinensamen ist identisch mit dem Lupinidin, welches aus den Samen der gelben Lupine isoliert ist. 
4. Den aus den schwarzen Lupinensamen hergestellten Alkaloiden, dem Lupinin und Lupinidin, kommen die von Banmert für die entsprechenden Alkaloide der gelben Lupine aufgestellten und von Berend bestätigten Formeln zu.

\section{Ueber die Alkaloide der perennierenden Lupine.}

(Lupinus polyphyllus.)

Von Dr. K. Gerhard.

Die ausdauernde Lupine, die als eine Hauptart der Lupinen anzusehen ist, umfasst eine grosse Anzahl von Unterarten (Agardh kennt deren 49$)^{1}$ ). Eine dieser Spezies ist die vielblätterige ausdauernde Lupine (Lupinus polyphyllus), deren Samen ich meinen Untersuchungen zu Grunde legte.

Lupinus polyphyllus ist die bei uns am meisten angebaute perennierende Lupine. Sie wird, wie die übrigen perennierenden Spezies, hauptsächlich als Zierpflanze gebaut, findet aber neuerdings auch Verwendung in der Landwirtschaft als Futterkraut und Stickstoffsammler. Einen gewissen Vorzug verdient nach $\mathrm{Kette}^{2}$ ) diese perennierende Lupine vor den anderen Lupinenarten aus dem Grunde, „weil sie, abweichend von allen einjährigen Lupinen, das Verpflanzen verträgt und dabei gegen Wurzelverwundungen ziemlich unempfindlich ist, daher also auch ohne Gefahr durch Behacken von Unkraut gereinigt werden kann".

Da Lupinus polyphyllus erst im zweiten Jahr zur Blüte kommt, habe ich aus den dieses Jahr gesäeten Samen noch keine blühende Pflanze erhalten; ich konnte daher die Identität der von mir verarbeiteten Samen mit denen der vielblätterigen perennierenden Lupine durch Vergleich beider Pflanzen bisher nicht nachweisen und muss mich auf den Identitätsnachweis der Samen beschränken.

Dieselben haben, übereinstimmend mit den von $\mathrm{Harz}^{3}$ ) beschriebenen Samen von Lupinus polyphyllus folgendes Aussehen: Die Samen sind variabel gefärbt; länglich oval, walzenförmig; ihre Hauptseite ist gewölbt, ihre Schmalseite gerundet. Sie sind $5 \mathrm{~mm}$ lang; 3,2 bis $3,5 \mathrm{~mm}$ hoch, 2,5 bis $2,8 \mathrm{~mm}$ dick, auf hell- oder dunkelgrauem, bis oliven- oder schwarzbraunem Grunde fein wolkig schwarz gefleckt oder marmoriert.

1) W. Kette, Lupinenbau, S. 2.

2) Ebendaselbst, S. 102.

3) Harz, Landwirtschaftl. Samenkunde. 